\title{
The Shape of Idealized Grain Boundaries
}

\author{
S. R. Coriell \\ Institute for Materials Research, National Bureau of Standards, Washington, D.C. 20234
}

(December 3, 1973)

\begin{abstract}
Mullins has treated a two-dimensional model of grain boundary motion in which each point on the boundary moves toward its center of curvature with a speed proportional to its curvature. For boundaries which preserve shape under uniform magnification, an integral representation of the boundary shape is found. We then obtain several analytic results from approximate evaluations of the integral.
\end{abstract}

Key words: Boundary shape; curvature; grain boundary.

The motion of an idealized grain boundary in two dimensions has been treated by Mullins [1]. ${ }^{1}$ In this model, a given point on the grain boundary moves towards its center of curvature with a speed that is proportional to the curvature. Recently Sun and Bauer [2] have extended the Mullins treatment by numerically solving the differential equation for the boundary shape for the case of boundaries which are invariant under magnification. They have used the results to measure grain boundary mobilities [3].

The shape of a boundary which is invariant under magnification is given by

$$
r(\theta, t)=R(\theta) T(t)
$$

where $r$ and $\theta$ are polar coordinates and $t$ is the time. $T(t)=\left[T^{2}(0)+2 c t\right]^{1 / 2}$, where $c$ is a separation constant and $R(\theta)$ satisfies the nonlinear differential equation

$\left\{R^{2}+2 R_{\theta}^{2}-R R_{\theta \theta}\right\} /\left\{R^{2}\left[R^{2}+R_{\theta}^{2}\right]\right\}=-(c / k)$.

Here, the subscript $\theta$ denotes differentiation with respect to $\theta$ and $k=M \sigma$ where $M$ is a mobility and $\sigma$ is the boundary free energy.

The purpose of this note is to obtain some analytic results for $R(\theta)$; in particular, the solution of eq (2) can be reduced to a single quadrature. Following standard techniques [4], we find

$$
\begin{gathered}
\theta(R)-\theta_{0}=\int_{R_{0}}^{R} d \alpha \alpha^{-1}\left\{C_{1} \alpha^{2}\right. \\
\left.\exp \left(c \alpha^{2} / k\right)-1\right\}^{-1 / 2},
\end{gathered}
$$

\footnotetext{
${ }^{1}$ Figures in brackets indicate the literature references at the end of this paper.
}

where $\theta\left(R_{0}\right)=\theta_{0}$ and $C_{1}$ is an integration constant. Sun and Bauer consider that a free surface acts as a mirror plane; thus for a free surface along the ray $\theta=\theta_{0}$ we take as an additional boundary condition $R_{\theta}=0$ at $R_{0}$. Introducing the change of variable

$$
\begin{aligned}
& \eta=\left(\alpha^{2} / R_{0}^{2}\right)-1, \text { eq }(3) \text { becomes } \\
& \qquad \begin{array}{r}
\theta(R)-\theta_{0}=\frac{1}{2} \int_{0}^{\rho} d \eta(1+\eta)^{-1}\{(1+\eta) \\
\quad \exp (b \eta)-1\}^{-1 / 2},
\end{array}
\end{aligned}
$$

where $\rho=\left(K^{2} / R_{0}^{2}\right)-1$ and $b=(c / k) R_{0}^{2}$. The boundary shapes for different values of $b$ have been discussed by Mullins. Figure 3 of reference [1] and figure 1 of reference [2] illustrate boundary shapes for various values of $b$. In reference [1], $R_{0}=3 / 2$ and $\theta_{0}=\pi / 2$ and in reference [2], $\theta_{0}=0$ and $\alpha$ and $f(\alpha)$ are equal to our $\theta(\infty)-\theta_{0}$ and $b$, respectively. From the equation for $T(t)$, we see that $b>0$ yields solutions which increase in size with time while $b<0$ gives solutions which shrink toward the origin $r=0$. For example, from eq. (2) it is clear that $b=-1$ is a circle of radius $R_{0}$; eq (4) is singular in this case since when $R(\theta)=R_{0}, \theta \neq \theta(R)$. The case $b=0$ corresponds to a straight line. For $b>0$, Sun and Bauer [2] solved numerically a differential equation, equivalent to eq (2), to obtain the boundary shape. For $b>0, R$ ranges from $R_{0}$ to infinity. For $b<0, R$ ranges

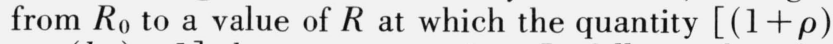
$\exp (b \rho)-1]$ becomes negative. It follows that for $-1<b<0, R$ is greater than $R_{0}$ while for $b<-1, R$ is less than $R_{0}$.

Although it does not appear possible to evaluate the right-hand side of eq (4) analytically, we can obtain some approximate analytic results. For values $|b \rho|$ 
$=\left|\left(b / R_{o}^{2}\right)\left(R^{2}-R_{o}^{2}\right)\right|<<1($ and $b \neq-1)$, we have $(1+\eta)$ $\exp (b \eta)-1 \cong(1+b) \eta\{1+(b \eta)(1+b / 2) /(1+b)\}$ and integration of eq (4) gives

$\cos \left\{2\left(1-b^{2} / 2\right)^{1 / 2}\left(\theta-\theta_{0}\right)\right\}$

$$
=\left\{1-\rho\left(1-b-b^{2}\right) /(1+b)\right\} /\{1+\rho\} .
$$

When $b>>1$, the only contribution to the integrand occurs for $\eta$ near zero. We can than approximate the integrand by $\{\exp (b \eta)-1\}^{-1 / 2}$. Upon integration and rearrangement, we obtain

$$
R=R_{0}\left\{1-(2 / b) \ln \cos \left[b\left(\theta-\theta_{0}\right)\right]\right\}^{1 / 2}
$$

We see that $\theta-\theta_{0}=\pi / 2 b$ when $R$ is infinite. Thus, eq (6) gives the shape of a grain boundary which makes a very small angle with a free surface.

Finally, for $R$ infinite, we obtain an approximate analytic formula for $\theta(\infty)-\theta_{0}$ as a function of $b$. We rewrite the integrand of eq (4) in the form $[(1+b) \eta]^{-1 / 2}[1+\eta]^{-1} \exp [-b \eta / 2][1+\{1-b \eta$ $\left.\left.-e^{-b \eta}\right\} /\{\eta(1+b)\}\right]^{-1 / 2}$. Expanding the last factor in powers of $b \eta$, yields

$$
\begin{aligned}
{\left[1+\left\{1-b \eta-e^{-b \eta}\right\} /\{\eta(1+b)\}\right]^{-1 / 2} } & \\
& =1+\alpha_{1} \eta+\alpha_{2} \eta^{2}+\ldots .
\end{aligned}
$$

where $\alpha_{1}=b^{2} / 4(1+b)$ and $\alpha_{2}=\left\{b^{3 / 96}(1+b)\right\}\{[9 b /$ $(1+b)]-8\}$. The resulting integrals can be evaluated in terms of error functions to give

$$
\begin{aligned}
\theta(\infty)-\theta_{0}=\{\pi / 2 b(1+b)\}^{1 / 2} & \left\{g+\alpha_{1}(1-g)\right. \\
+ & \left.\alpha_{2}\left(g-1+b^{-1}\right)\right\},
\end{aligned}
$$

where $g=(\pi b / 2)^{1 / 2} \exp (b / 2)$ erfc $\left[(b / 2)^{1 / 2}\right]$. The $\alpha_{2}$ term is small compared to the leading term. Thus, eq (7) provides an analytic representation of the numerical results for $b$ as a function of $\theta(\infty)-\theta_{0}$, which are given in figure 2 of reference [2]. It is perhaps surprising that eq (7) is a good approximation for all values of $b$, since it is based on an expansion in powers of $(b \eta)$. However, when $b \eta$ is not small, the integrand is very small due to the exponential term and makes a negligible contribution to the integral. For $b<<1$, eq (7) yields

$$
\theta(\infty)-\theta_{0}=\frac{\pi}{2}\left[1-(2 b / \pi)^{1 / 2}\right]
$$

and for

$b \rightarrow \infty, \theta(\infty)-\theta_{0}=b^{-1}$

$$
(\pi / 2)^{1 / 2}[1+(1 / 4)+(1 / 32)]=1.61 / b,
$$

which is in reasonable agreement with the result $(\pi / 2 b)$ obtained from eq (6). As an example of an intermediate value of $b$, we find that for $b=7.1, \theta(\infty)-\theta_{0}=$ $10.0^{\circ}$ in agreement with Sun and Bauer.

\section{References}

[1] Mullins, W. W., J. Appl. Phys. 27,900 (1956).

[2] Sun, R. C., and Bauer. C. L., Acta Met. 18, 635 (1970).

[3] Sun, R. C., and Bauer. C. L., Acta Met. 18, 639 (1970).

[4] Murphy, G. M., Ordinary Differential Equations and Their Solutions (Van Nostrand, Princeton, N. J. 1960), p. 159-161 and p. 26.

(Paper 78A2--808) 